\title{
Immunohistochemical Evaluation of Calretinin and Cytokeratin-19 in Odontogenic Keratocyst and Ameloblastoma: A Retrospective Study
}

\author{
${ }^{1}$ Vipul Mohan Pawar, ${ }^{2}$ Shilpa Patel, ${ }^{3}$ Jigna Pathak, ${ }^{4}$ Niharika Swain, ${ }^{5}$ Rashmi Hosalkar, ${ }^{6}$ Janaki lyer
}

\begin{abstract}
The odontogenic epithelial remnants, i.e. cell rests of Serre and Malassez, are formed from dental lamina and Hertwig's epithelial root sheath respectively, may proliferate and have role in pathogenesis of odontogenic cysts and tumors. Odontogenic keratocyst (OKC) is the most common and aggressive cyst of the dental lamina origin. Ameloblastoma, the second most common odontogenic tumor (OT), is a clinically benign and locally invasive polymorphic neoplasia. Differentiation of OKC from ameloblastoma sometimes poses a diagnostic dilemma, thus necessitating the need to differentiate between the two (especially unicystic ameloblastoma and OKC). Calretinin, a calcium binding protein, functions as a calcium buffer and a regulator of apoptosis. Some studies have shown its expression in parakeratinized OKC, unicystic and solid ameloblastoma, but not in other OTs. Calretinin may thus provide a better understanding of the biological behavior and tumorogenesis of ameloblastoma. Cytokeratin (CK)-19 is a type I cytokeratin, has been found to be a reliable marker of epithelial differentiation. The intense expression of CK-19 is useful for identification of odontogenic epithelial components, thus suggesting their potential for proliferation to form epithelial odontogenic cysts and tumors. The aim of this study is to evaluate calretinin and CK-19 in OKC and ameloblastoma. For this retrospective study, 20 formalin fixed paraffin embedded tissue samples of histopathologically proven OKC and ameloblastoma each, retrieved from the department of oral pathology was used. The results will be evaluated by using immunohistochemical analysis.
\end{abstract}

Keywords: Ameloblastoma calretinin, Cytokeratin, Immunohistochemistry, Orthokeratinized odontogenic cyst.

How to cite this article: Pawar VM, Patel S, Pathak J, Swain N, Hosalkar R, lyer J. Immunohistochemical Evaluation of Calretinin and Cytokeratin-19 in Odontogenic Keratocyst and Ameloblastoma: A Retrospective Study. J Contemp Dent 2015;5(2):98-103.

\section{Source of support: Nil}

Conflict of interest: None

\section{INTRODUCTION}

The dental lamina that forms the enamel organs as well as Hertwig's epithelial root sheath (HERS), which forms the

\footnotetext{
${ }^{1,5,6}$ Postgraduate Student, ${ }^{2}$ Professor and Head

${ }^{3}$ Professor, ${ }^{4}$ Lecturer

1-6Department of Oral Pathology, MGM Dental College and Hospital, Navi Mumbai, Maharashtra, India

Corresponding Author: Vipul Mohan Pawar, Postgraduate Student Department of Oral Pathology, MGM Dental College and Hospital Navi Mumbai, Maharashtra, India, Phone: 9822387600 , e-mail: vipul242428@gmail.com
}

roots of the teeth disintegrates and gives rise to odontogenic epithelial remnants called cell rests of Serre and cell rests of Malassez respectively. Although a vast majority of these epithelial residues persist throughout life in an inactive state, some of these are triggered by hitherto unknown mechanisms to proliferation and, thus, produce a variety of pathogenesis comprising of odontogenic cysts and tumors. ${ }^{1}$

Odontogenic keratocyst $(\mathrm{OKC})$ is one of the common odontogenic cysts arising from the dental lamina. It may grow to a large size before it manifests clinically and it has a striking tendency to recur. ${ }^{2}$ Various studies are performed to ascertain the aggressive nature of OKC, thus considering OKC as a benign cystic neoplasm. ${ }^{3}$ Odontogenic tumors (OTs) are a group of lesions arising from the tooth producing apparatus or its remnants. They may originate from odontogenic epithelium and/or ectomesenchyme with varying degree of inductive tissue interaction. ${ }^{4,5}$ Ameloblastoma is the second most common odontogenic neoplasm. World Health Organization (WHO) defines ameloblastoma as 'a locally invasive, polymorphic neoplasia that often has a follicular or plexiform pattern in a fibrous stroma'. ${ }^{6}$ Ameloblastoma can also be classified into solid/multicystic (SMA) and unicystic ameloblastoma (UA). The biological behavior, aggressiveness, treatment and prognosis between SMA and UA varies, thus necessitating the need to differentiate between the two.

Differentiation of OKC from ameloblastoma sometimes poses a diagnostic dilemma, thus compelling the need to differentiate between the two (especially UA and $\mathrm{OKC}$ ). Various immunohistochemical studies have been carried out in OKC and ameloblastomas. Of these markers, calretinin and cytokeratin (CK)-19 have proven to be important in differentiating and determining the proliferative potential of OKC and ameloblastoma. Calretinin, a calcium binding protein with a molecular weight of 29 kilodalton $(\mathrm{kDa})$, is a member of EF-hand proteins. In humans, it is enclosed by CLAB-2 gene. The exact biological function of calretinin remains unknown, but possible roles as calcium buffer and/or calcium sensor and regulator of apoptosis have been postulated. ${ }^{6,7}$ Intracellular $\mathrm{Ca}^{2+}$ ions are considered to be important second 
messengers intervening in several cellular processes, including proliferation and differentiation. Previous study in rats have demonstrated calretinin expression in neural element of tooth pulp, periodontal ligament and viscerosensory nerve fiber of oral and pharyngeal tissues as well as in odontogenic epithelium during odontogenesis in molar tooth germs at early cap stage. As the tooth development progresses, the intensity of reactivity increases from weak to intense in the late bell stage. ${ }^{4}$ Adriano Piattelli et al 2003 conducted a study to demonstrate calretinin expression in OKC and concluded that calretinin showed its presence in some cases of parakeratinized OKC. It is also reported in UA and SMA, however, this protein has not been demonstrated in other OTs. Calretinin, as an immunohistochemical marker may thus provide a better understanding of the biological behavior, influencing factors and tumorogenesis of ameloblastoma.

The keratins are intermediate filament proteins responsible for structural integrity of epithelial cells and are subdivided into cytokeratin and hair keratin. Cytokeratin-19 is a type-Ikeratin consisting of acidic proteins which are arranged in pairs of heterotypic keratin chains. In humans, it is encoded by keratin gene (KRT)-19 gene. It is specifically found in the periderm, the transiently superficial layer that envelops the developing epidermis. ${ }^{11}$ Cytokeratin plays a role in tissue specialization and function to maintain the overall structural integrity of epithelial cells. They are expressed in simple epithelium and basal layer of nonkeratinizing stratified squamous epithelium. It has been found to be a useful marker of epithelial differentiation and proliferation ${ }^{9,12,13}$ which is directly applicable to the characterization of OKC and ameloblastoma. The overexpression of CK-19 is useful for identification of odontogenic epithelial components, in evaluating the various types of epithelial odontogenic cysts and tumor and their potential for proliferation.

\section{MATERIALS AND METHODS}

\section{Case Selection}

Formalin-fixed paraffin-embedded tissue blocks of orthokeratinized odontogenic cyst (OOC), OKC and ameloblastoma (unicystic and multicystic variants), were retrieved from the archives of Department of Oral and Maxillofacial Pathology, MGM Dental College and Hospital.

\section{Immunohistochemistry ${ }^{14}$}

Formalin-fixed paraffin-embedded tissue blocks were cut into $4 \mathrm{~m}$ thick sections and placed on organosilanepretreated slides. Immunohistochemical staining was performed using the primary antibody for calretinin and CK-19 using supersensitive one-step polymer-HRP technique (Biogenex Life Sciences, San Ramon, CA, USA). The sections were deparaffinized and rehydrated through xylene and descending grades of alcohol, respectively. Antigen retrieval was carried out using commercial microwave antigen retrieval system where the sections were immersed in $10 \mathrm{mM}$ sodium citrate buffer $(\mathrm{pH} 6.0)$ at 800,420 , and $320^{\circ} \mathrm{F}$ for three cycles of 10 minutes each (EZ-Retriever System, Biogenex Life Sciences). After rinsing in tris buffer ( $\mathrm{pH}$ 7.2), the sections were incubated with $3 \%$ hydrogen peroxide in water for 15 minutes to block the endogenous peroxidase activity. This was followed by a power block for 10 minutes at room temperature to block any nonspecific antigenic sites. The sections were then incubated with optimally prediluted rabbit polyclonal antibody against calretinin and CK-19 (Biogenex, CA, USA) for 30 minutes at room temperature in a moist chamber. After washing with tris buffer ( $\mathrm{pH}$ 7.2) for 5 minutes, the sections were then incubated with super enhancer for 20 minutes at room temperature, followed by secondary antibody incubation with one-step polymer-HRP reagent for 30 minutes at room temperature in a moist chamber. The slides were counterstained with Mayer's hematoxylin for 3 minutes, subsequent to which sections were dehydrated, cleared, and mounted with dibutyl phthalate xylene (DPX) evaluation of slides. The evaluation of the stained slides was carried out using a binocular research microscope under 100 and 400× magnification.

\section{RESULTS}

The study group comprised of groups I and II, each having 20 cases. Group 1 consisted of 8 cases of OOC and 12 cases of OKC while group II consisted of 15 cases of SMA and 5 cases of UA. The samples were subjected to immunohistochemical procedures, as described in the section of materials and methods, to detect calretinin and CK-19 positivity. The calretinin and CK-19 positive cells were indexed according to the staining criteria by evaluating immunopositivity/staining percentage and staining intensity. Staining percentage was considered as focal $(<50 \%)$ and diffuse $(>50 \%)$, while staining intensity as, 0 -no staining, 1 -staining observed at $400 \times$ (weak), 2-staining observed at 100× (moderate), 3-staining observed even at $40 \times$ (strong). The data obtained was presented using descriptive statistics, such as mean, standard deviation, standard error and confidence interval. Also the data was presented using appropriate graphs and charts. All collected data were entered into Statistical Package for the Social Sciences (SPSS) 16.0 worksheet. Further analysis was performed using statistical test, such as Chi-square test. A significance level of 0.05 
was applied to decide the statistical significance of any statistic obtained. In group I, out of the 20 cases, 8 cases were of OOC $(n=8)$ and 12 cases were of OKC $(n=12)$. In group II, among the 20 cases of ameloblastoma $(n=20)$, 15 were of solid variant $(n=15)$ and five were UA $(n=5)$.

Table 1 showed comparison of staining percentage and staining intensity of calretinin expression in OOC, OKC, SMA and UA. In OOC and OKC, $(n=7)$ showed negative immunoreactivity out of which six were OOC and one was OKC, $(n=11)$ showed focal immunoreactivity out of which two were OOC and nine were OKC and $(n=2)$ showed diffuse immunoreactivity of which all were OKC. In ameloblastoma $(n=6)$ showed negative immunoreactivity out of which five were SMA and one was UA, $(n=14)$ showed focal immunoreactivity out of which 10 were SMA and four were UA.

In OOC and OKC, $(n=7)$ showed negative staining, out of which six were OOC and one was OKC, $(n=2)$ showed weak staining and all were $\mathrm{OKC},(\mathrm{n}=6)$ showed moderate staining, out of which one was OOC and five were OKC and ( $\mathrm{n}=5$ ) showed strong staining, out of which one was OOC and four were OKC. In ameloblastoma, $(n=6)$ showed negative staining, out of which five were SMA and one was UA, $(n=4)$ showed weak staining, out of which three SMA and one was UA, $(n=7)$ showed moderate staining out of which four were SMA and three were UA and $(n=3)$ showed strong staining of which all were SMA (Figs $1 \mathrm{~A}$ and 2A).

Table 2 showed comparison of immunopositivity and staining intensity of CK-19 expression in OOC, OKC, SMA and UA. In OOC and OKC, $(\mathrm{n}=4)$ showed negative immunoreactivity and all were OOC, $(n=6)$ showed focal immunoreactivity out of which two were OOC and four were OKC and $(n=10)$ showed diffuse immunoreactivity out of which two were OOC and eight were OKC. In ameloblastoma $(n=12)$ showed focal immunoreactivity out of which eight were SMA and four were UA and $(n=8)$ showed diffuse immunoreactivity out of which six were SMA and two were UA.

In OOC and OKC, $(\mathrm{n}=4)$ showed negative staining and all were OOC, $(n=4)$ showed moderate staining, out of which one was OOC and three were OKC and $(n=12)$ showed strong staining, out of which three were OOC and nine were OKC. In ameloblastoma, $(n=1)$ showed weak staining and it was SMA, $(n=9)$ showed moderate

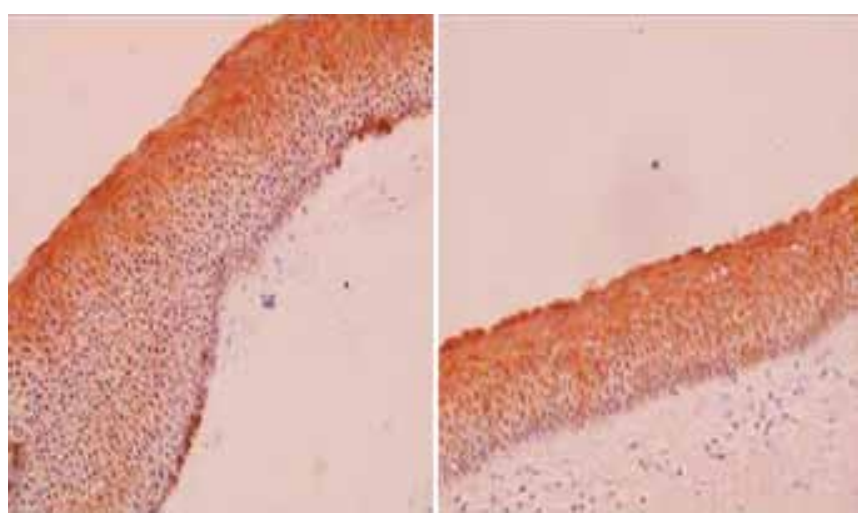

Figs $1 A$ and $B$ : Diffuse immunoexpression of calretinin $(A)$ and CK-19 (B) in OKC (400x magnification)

staining out of which seven were SMA and two were UA and $(n=10)$ showed strong staining of which seven were SMA and three were UA (Figs 1B and 2B).

\section{DISCUSSION}

Various pathologies like odontogenic cysts and tumors commonly affect the jaw bones. Odontogenic keratocyst is a clinicopathologically distinct form of odontogenic cyst that is noted for its aggressiveness and high recurrence rate. Latest WHO classification has suggested that $\mathrm{OKC}$ is to be considered a neoplasm because of its growth pattern and high recurrence rate. Ameloblastoma is the second most common odontogenic neoplasm. World Health Organization defines ameloblastoma as 'a locally invasive, polymorphic neoplasia that often has a follicular or plexiform pattern in a fibrous stroma'. Ameloblastoma can also be classified into SMA and UA and DA. Odontogenic keratocyst is an aggressive cyst with neoplastic behavior while ameloblastoma is a benign, locally aggressive epithelial odontogenic tumor that has the potential to become malignant and produce metastasis to distant sites, such as lungs and kidneys. Unicystic ameloblastoma, however, is a variant with better prognosis and less aggressive behavior. The biological behavior, aggressiveness, treatment and prognosis between OKC and ameloblastoma varies, thus necessitating the need to differentiate between the two. The purpose of this study was to evaluate the immunopositivity of calretinin and CK-19 in group I (OKC and OOC) and group II (SMA and UA) and to establish the possible role of calretinin and CK-19 in the biological behavior of these lesions.

Table 1: Comparison of staining percentage and staining intensity of calretinin expression in OOC, OKC, SMA and UA

\begin{tabular}{|c|c|c|c|c|c|c|c|c|}
\hline \multirow[b]{2}{*}{ Groups } & \multirow[b]{2}{*}{ No. of cases } & \multicolumn{3}{|c|}{ Staining percentage } & \multicolumn{4}{|c|}{ Staining intensity } \\
\hline & & Negative & Focal & Diffuse & 0 (negative) & 1 (weak) & 2 (moderate) & 3 (strong) \\
\hline OOC & 8 & 6 & 2 & 0 & 6 & 0 & 1 & 1 \\
\hline OKC & 12 & 1 & 9 & 2 & 1 & 2 & 5 & 4 \\
\hline SMA & 15 & 5 & 10 & 0 & 5 & 3 & 4 & 3 \\
\hline UA & 5 & 1 & 4 & 0 & 1 & 1 & 3 & 0 \\
\hline
\end{tabular}


Immunohistochemical Evaluation of Calretinin and Cytokeratin-19 in Odontogenic Keratocyst and Ameloblastoma

Table 2: Comparison of staining percentage and staining intensity of CK-19 expression in OOC, OKC, SMA and UA

\begin{tabular}{|c|c|c|c|c|c|c|c|c|}
\hline \multirow[b]{2}{*}{ Groups } & \multirow[b]{2}{*}{ No. of cases } & \multicolumn{3}{|c|}{ Staining percentage } & \multicolumn{4}{|c|}{ Staining intensity } \\
\hline & & Negative & Focal & Diffuse & $O$ (negative) & 1 (weak) & 2 (moderate) & 3 (strong) \\
\hline OOC & 8 & 4 & 2 & 2 & 4 & 0 & 1 & 3 \\
\hline OKC & 12 & 0 & 4 & 8 & 0 & 0 & 3 & 9 \\
\hline SMA & 15 & 0 & 9 & 6 & 0 & 1 & 7 & 7 \\
\hline UA & 5 & 0 & 3 & 2 & 0 & 0 & 2 & 3 \\
\hline
\end{tabular}
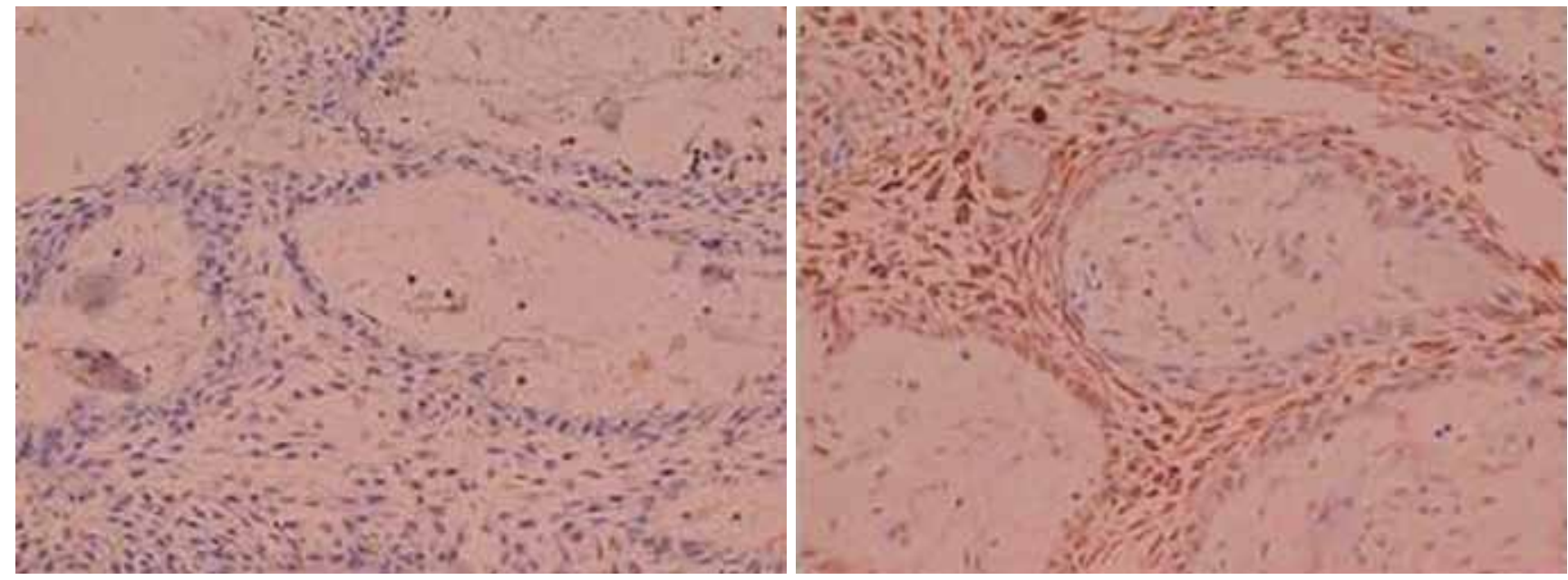

Figs 2A and B: Focal immunoexpression of calretinin (A) and diffuse expression of CK-19 (B) in ameloblastoma (400× magnification)

In contrast to our study, Coleman et $\mathrm{al}^{7}$ showed no immunopositive epithelial staining in any cystic lesions (22 OKC). Piattelli et $\mathrm{al}^{15}$ observed that all OOC were negative to calretinin, however, $66.66 \%$ of OKC showed positivity to calretinin in the parabasal and intermediate layers of epithelial lining, while other layers were negative which was in accordance with our study. Scharfetter et $\mathrm{al}^{15}$ stated that the epithelium of the keratocyst showed a higher rate of proliferation than the other cyst and positivity could be related to the increased mitotic value. Gotz et $\mathrm{l}^{15}$ observed that calretinin was found in rapidly proliferating cells. A similar retrospective study by Altini et $\mathrm{al}^{8}$ found calretinin immunopositivity in $81.5 \%$ of UA and $93.5 \%$ of solid ameloblastoma. Unicystic ameloblastoma showed clumped, diffuse, intense cytoplasmic and nuclear staining in several layers of the more superficial and luminal epithelial cells, whereas stellate reticulumlike epithelium produced aband-like appearance. In solid ameloblastoma, staining was diffusely distributed in to the stellate reticulum-like epithelium while only one case showed focal positivity in basal cells. Alaeddini et $\mathrm{al}^{4}$ found that calretinin immunoreactivity was positive only for ameloblastoma when compared to other odontogenic tumors, stating that this protein may have a role in the transition of the dental lamina remnants to ameloblastoma. They hypothesized that calretinin may be one of the factors responsible for the differences between this aggressive neoplasm and other odontogenic tumors studied. Altini et $\mathrm{al}^{7}$ indicated the inverse relationship of the calretinin expression. The cases of UA which were lined by typical ameloblastic epithelium showed no or little calretinin immunopositivity, i.e. the better the differentiation of the epithelium was, lesser the expression of calretinin. Hence, they suggested that calretinin expression in some cells varied according to their metabolic activity and may be lost when this activity changes. The altered expression of calretinin in developing tooth as well as in odontogenic cyst and neoplasm indicates its orthologic and pathologic role in odontogenesis respectively. Some studies have shown its varied expression in ameloblastoma and in lesser extent in OKC, but not in other OTs. Previous retrospective studies ${ }^{7,8}$ on calretinin expression in OOC and OKC, observed no or little expression while 80 to $100 \%$ immunoreactivity was seen in ameloblastoma. This was in contrast to our observation, where little to nil immunoexpression was seen in OOC, whereas focal immunoexpression was seen in $66.66 \%$ of OKC in suprabasal and superficial cell layer. Also, focal immunoexpression was seen in $66.66 \%$ of SMA in peripheral and stellate reticulum like cells and $80 \%$ of UA in superficial layer. The varied expression of calretinin in neoplastic ameloblast like cells, in contrast to developing ameloblasts in normal teeth, is noteworthy and warrants further investigation of cyto-differential transition from normal to neoplastic tissue. If there is any possible relation between calretinin expression in odontogenic 
epithelium and its neoplastic transformation, then calretinin could be used as an early marker to predict the tendency of neoplastic change of odontogenic epithelium. In our study, since calretinin immunoexpression was seen in $66.66 \%$ of OKC and SMA, OKC could also be considered as a benign neoplasm as suggested by some authors. ${ }^{16}$ Calretinin expression in OKC and ameloblastoma further highlights the aggressive and neoplastic nature of OKC with respect to OOC. This mandates the need to differentiate OOC from OKC. Since the sample size in our study could not statistically highlight this, further research with more sample size is warranted to support our observation.

This is in accordance with a study by Hayakawa et al who found all or most of the three layers (basal, spinous and cornified) were positive for CK-17 and 19. A study by Aragaki et al evaluated the immunohistochemical profile of the keratin expression in keratocystic odontogenic tumor (KCOT) and OOC. Orthokeratinized odontogenic cyst expressed keratin 1,2 and 10 suggesting differentiation toward normal epidermis, while KCOT expressed keratin 4, 13, 17 and 19, were suggestive of a mucosal squamous epithelium. Similar to our study, Chaitanya Babu, ${ }_{13}^{13}$ observed CK-19 expression in both basal cells and stellate reticulum-like cells in ameloblastoma. They stated that intense expression of CK-19 suggested the potential for proliferation in ameloblastoma. Kanth et $\mathrm{al}^{17}$ found strong positivity of CK-19 in the peripheral columnar cells and weak positivity in the central stellate reticulum type cells in ameloblastoma. Kumamoto et al observed diffuse immunopositivity for CK-19 in all cell layers of ameloblastomas. MN Ong'uti et al, found that all 39 cases of ameloblastoma showed CK-19 expression (75-100\%) in tumor epithelium including basal, suprabasal and central cells and the staining was wide spread throughout the tumor. Cytokeratins are universal indicator of epithelium specificity; their altered expression was evident in various benign, hyperplastic and neoplastic lesions. Few researches on CK-19 expression in OOC and OKC observed no or little expression, in contrast to our observation where mild expression was seen in 50\% of OOC (4 of 8 cases) only in superficial layer, while $100 \%$ of OKC (all 12 cases) showed intense expression in suprabasal and superficial cell layer. In embryological odontogenesis, positive immunoreactivity of CK-19 in preameloblast and secretory ameloblast indicates its association with secretory differentiation. In odontogenic tumor, especially ameloblastoma, previous studies claimed 100\% overexpression of CK-19 in suprabasal and central cells. However, in our study, $100 \%$ cases of ameloblastoma (SMA and UA) showed increased immunoreactivity. The observed overexpression of CK-19 in the transformed odontogenic epithelium suggested the proliferative potential and delay in commitment to terminal differentiation of the same. Since a number of studies observed that the expression of CK-19 was restricted to the basal cell layer of nonkeratinized epithelium, altered expression of CK-19, i.e. expression in suprabasal cells, could be considered to be indicator of dysplastic changes. ${ }^{12}$ Thus, it can be hypothesized that consistent overexpression of CK-19 in suprabasal cells of OKC and ameloblastoma including UA, unlike in the superficial layers of OOC, can be attributed to their proliferative potential and well established aggressive biological behavior.

\section{CONCLUSION}

Though the diagnosis of various odontogenic cysts and tumors exclusively count on histopathological examination, various crucial factors, like detection of neoplastic or dysplastic changes and assessment of proliferation potential, cannot bejudged with this conventional method. With the advent of IHC, a novel technique, we may overcome the aforementioned limitations, that may assist to determine the transition from normal to neoplastic tissue. Though this skill is still within grey zones, we propose and emphasize the use of markers like calretinin and CK-19 to establish their potential for predicting the biological behavior and proliferation of OKC and ameloblastoma, thereby affecting the treatment and prognosis. Although the probable association between the expression of these markers and the growth potential of these aggressive lesions could not be statistically established through this study, owing to limited sampling, yet, we would like to propose further exploration into this innovative system.

\section{REFERENCES}

1. Garant P. Oral cells and tissues, edition illustrated;2003. p.1-2.

2. Shear M, Paul M. Cyst of the oral and maxillofacial regions. 4th ed. p. 28-30.

3. Shear M. The aggressive nature of the odontogenic keratocyst: is it a benign cystic neoplasm? Part 3 immunocytochemistry of cytokeratin and other epithelial cell markers. Oral Oncol 2002;38(38):407-415.

4. Alaeddini M, Etemad-Moghadam S. Comparative expression of calretinin in selected odontogenic tumours: a possible relationship to histogenesis. Histopathol 2008;52(3):299-304.

5. Reichart $\mathrm{P}$, Philipsen $\mathrm{H}$. Odontogenic tumors and allied lesions 2004;17-18.

6. Saify F, Sharma N. Basal cell ameloblastoma: a rare case report and review of literature. JOMPJ 2010;1(1):1.

7. Coleman H, Altini M, Doglioni C, Favia G, Maiorano E. Use of calretinin in the differential diagnosis of unicystic ameloblastoma. Histopathol 2001;38(4):312-317. 
8. Altini M, Coleman H, Doglioni C, Favia G, Maiorano E. Calretinin expression in ameloblastoma. Histopathol 2000;37(1):27-32.

9. Kumamoto H, Yoshida M, Ooya K. Immunohistochemical detection of amelogenin and cytokeratin 19 in epithelial odontogenic tumors. Oral Diseases 2001;7(3):171-176.

10. Stoll C, Stollenwerk C, Riediger D, Mittermayer C, Alfer J. Cytokeratin expression patterns for distinction of odontogenic keratocysts from dentigerous and radicular cysts. J Oral Pathol Med 2005;34(9):558-564.

11. Chu P, Weiss L. Keratin expression in human tissues and neoplasms. Histopathol 2002;40(1):403-439.

12. Chatterjee S. Cytokeratins in health and disease. JOMP 2012; 3(1):198-202.
13. Chaitanya Babu N, Dawra G, Sindura CS. Immunohistochemical evaluation of Bcl2 and Cytokeratin 14 and Cytokeratin 19 in ameloblastoma. IJCD 2010;1(1):36-39.

14. Bancroft J, Gamble M. Theory and practice of histological techniques. 6th ed. Churchill Livingstone Elsevier. p. 432-472.

15. Piattelli A, Lezzi G, Rubini C. Calretinin expression in odontogenic cysts. J Am Asso Endo 2003;29:6.

16. Shear M, Paul M. Speight, cyst of the oral and maxillofacial regions. 4th ed; 2006 Oct.

17. Kanth KS, Kumar1 TD, Kumar AR. Immunohistochemical analysis of dentigerous cyst and ameloblastoma using cytokeratin 19 and 14, p53, p63 and ki-67. SRM J Res Dent Sci 2012 Oct-Dec;3(4):4. 